\title{
SINGLE-CRYSTAL DIAMOND NEMS DEVICES FOR THE STUDY OF COLOR CENTERS
}

\author{
Young-Ik Sohn $\eta^{\dagger}$, Srujan Meesala ${ }^{\dagger}$, Michael J. Burek, Haig A. Atikian and Marko Lončar* \\ John A. Paulson School of Engineering and Applied Sciences, Harvard University, 29 Oxford Street, Cambridge, \\ MA 02138, USA \\ $\dagger$ These authors contributed equally to this work.
}

\begin{abstract}
We demonstrate fabrication methods for micro-/nanoelectromechanical systems (MEMS/NEMS) in single-crystal diamond chip. Such devices can be used to study embedded color centers, such as the nitrogen vacancy (NV) or the silicon vacancy $(\mathrm{SiV})$. Color centers have discrete electronic and spin energy levels, which can be controlled by engineering the amount of strain in the diamond lattice. These strain fields can be effectively controlled using conventional MEMS/NEMS devices. As a specific demonstration, we show the tuning of $\mathrm{SiV}$ electronic energy levels via static actuation. The device design process accounting for various fundamental and experimental constraints is discussed.
\end{abstract}

\section{INTRODUCTION}

Solid-state qubits have been extensively studied for the last few decades in the field of quantum information science and technology (QIST). Recently, color centers in single-crystal diamond (SCD), have attracted attention as solid-state qubit candidates. The most extensively studied color center is the nitrogen-vacancy (NV) center, whose ground state spin sublevels have long coherence time (on the order of milliseconds) at room temperature. Another color center, which has received renewed attention recently, is the silicon vacancy ( $\mathrm{SiV}$ ) center, due to its excellent optical properties, such as the large zero-phonon line at room temperature (approximately 70\%) and small inhomogeneous distribution.

These color centers possess discrete energy levels corresponding to electronic orbital states. The spin sublevels of these orbitals can be split to have different energies by the application of an external magnetic field. Such spin sublevels can be used to define a qubit. A qubit can be typically manipulated with external control fields such as a magnetic field (Zeeman effect) or an electric field (Stark effect).

Recently, it has been proposed that strain fields in the host lattice can be used to manipulate the quantum state of qubit [1]. Therefore, controlled generation of strain fields at the site of a target qubit, with a specific frequency and direction, has been of keen interest. The simplest approach is to use an external actuator, e.g. piezoelectric transducer, to induce static or dynamic motion [2-4]. External actuators, however, are typically bulky so that they are not compatible with solid-state qubit experiments in certain types of experiments. For example, in diamond color center studies optical control and read-out is necessary, and the sample needs to be placed under an (within a millimeter) objective lens. In the case of dynamic actuation, it can be conveniently achieved by attaching a piezo actuator externally and applying AC voltage [2, 4]. However, most commercially available piezo actuators for dynamic actuation have cut-off frequency near $1 \mathrm{MHz}$, limited by their mass.

To date, high-overtone bulk acoustic resonator (HBAR) [5] and surface acoustic wave (SAW) devices [6] operating at few $\mathrm{GHz}$ frequency have been fabricated on single-crystal diamond and used to manipulate quantum states of NV center. The two platforms mentioned above demonstrated resonant coupling of spin states and parametric coupling of excited orbital states to strain, respectively. These devices are built on the flat surface of bulk sample and typically have large mechanical mode volume. However, many quantum information applications require the strong interaction of single mechanical quanta (phonons) and qubits, and this can only be achieved with small mechanical mode volumes. The realization of MEMS/NEMS devices in single crystal diamond can help achieve very small mode volumes, and allow actuation and detection of mechanical modes with electrical signals. However, there are several challenges in the fabrication of these devices, particularly since they should be compatible with optics experiments on color centers. In this work, we demonstrate MEMS/NEMS devices fabricated on SCD, which can be applied to color center experiments. In particular, we discuss the application of one such device to $\mathrm{SiV}$ electronic level tuning via application of static strain.

(a)
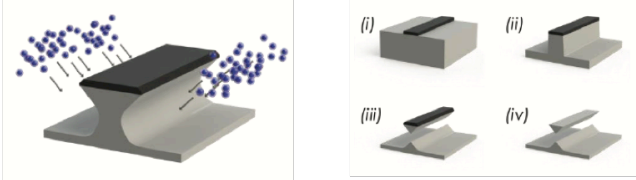

(b)

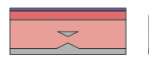

(i)

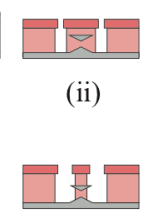

(ii)

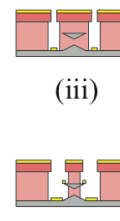

(iii)

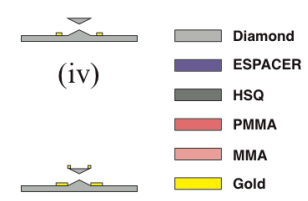

(iv)
Figure 1: (a) Illustration of angle-etching, through steps (i)(iv). (b,c) Fabrication steps of diamond nanobeams with (b) dielectrophoretic and (c) electrostatic actuation. Standard bilayer lift-off process is used. Fabrications steps are mostly the same between (b) and (c) except for the subtle difference in the pattern in (iii).

\section{RESULTS}

\section{Fabrication}

Currently, SCD can only be synthesized in the bulk form, which impedes the fabrication of free-standing nanostructures on them. To overcome such a challenge, in our earlier work, we reported a fabrication method for free-standing structures in SCD [7]. A Faraday cage is placed in the chamber during reactive-ion etching, which allows etching at an oblique angle. Illustration of our etching scheme (referred hereafter "angled-etching") is shown in Fig. 1(a). First, the mask pattern is formed by spinning e-beam resist (hydrogen silsesquioxane, (HSQ)) and writing with an ebeam lithography tool. The device is vertically etched down by reactive-ion etching (RIE), where oxygen plasma chemistry is applied [8]. The next step is angled-etching, in which a Faraday cage is placed inside the RIE chamber, and it alters the electrical
Solid-State Sensors, Actuators and Microsystems Workshop Hilton Head Island, South Carolina, June 5-9, 2016 
potential and the sheath. The environment in the chamber accelerates ions in the plasma at a range of oblique angles, eventually shaping free-standing structures with a triangular crosssection. The physics of angled-etching has been systematically studied with three different materials [9]. With proper design of the mask pattern, various free-standing structures can be made at the micro- and nano-scale. Devices demonstrated by this technique includes nanomechanical resonators [10], as well as more sophisticated devices like photonic crystal optical cavities [11].

For the fabrication of an on-chip actuator, a standard bi-layer lift-off process is performed to place electrodes around the resonator. In this work, we present two complementary approaches that result in different device configurations and driving mechanisms, suitable for color center experiments. In the first approach, based on dielectrophoretic actuation, electrodes can be placed on either side of diamond nanobeam in close proximity without physically contacting it. In the second approach, the electrodes are placed on the cantilever itself, and electrostatic actuation is performed. The fabrication process is illustrated in Fig. 1(b). First, diamond sample is spin-coated with polymethyl methacrylate (PMMA) multiple times, to ensure that the entire chip is covered with the resist. As a next step, pre-fabricated alignment markers are revealed by exposing the nearby area and developing the sample subsequently. (This step is not shown in Fig. 1 for simplicity, but it is important to achieve high precision alignment in the next step.) After the markers are cleared, a conductive layer (metal or ESPACER ${ }^{\mathrm{TM}}$ [12]) is formed on top of the PMMA layer to enhance the quality of the electron-beam lithography (EBL). Then electrode patterns are written and developed. After that, a quick oxygen plasma clean (20 sccm, $250 \mathrm{mTorr}, 100 \mathrm{~W}, 1 \mathrm{~min})$ can be performed (optional) for a cleaner pattern and surface quality. Finally, desired metals are evaporated on the patterned device and lift-off is performed at $80^{\circ} \mathrm{C}$ in Remover PG to finalize the device. Depending on the dimensions of the nanobeam, critical point drying may be used to prevent the device from snapping down to the substrate.

\section{Characterization}

Devices made by the process shown in Fig. 1(b) can be dynamically actuated through dielectrophoretic force [13]. SEM images of the devices are shown in Fig. 2(a) and (b). We have reported dielectrophoretic actuation of devices previously [14]. Resonantly driven motion of such devices is shown in Fig. 3(a) and (b), when RF voltage was applied across two electrodes on either side. The motion of the cantilever is optically read out by using optical interferometry [15]. Displacement of the nanobeam motion can be thermomechanically calibrated by measuring thermal fluctuations [16]. Fig. 3(a) and (b) correspond to the cantilever and doubly clamped beam in Fig. 2(a) and (b), respectively.

Dielectrophoretic actuation can be useful for resonant driving when high mechanical quality factor $(Q)$ is desired. However, the
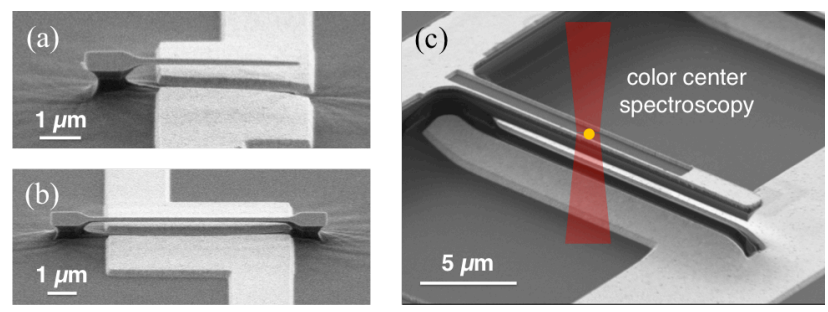

Figure 2: SEM images of diamond nanobeams with $(a, b)$ dielectrophoretic and (c) electrostatic actuation.
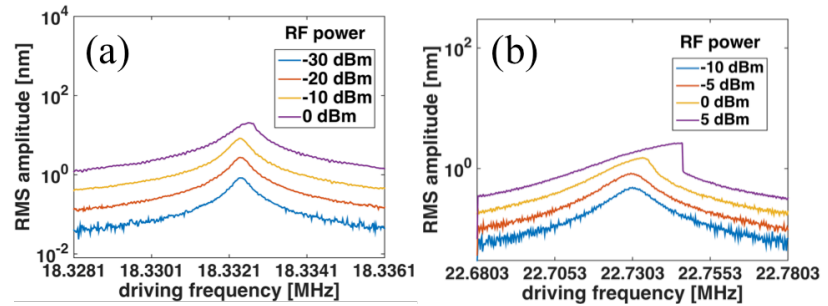

Figure 3: Resonantly driven motion of (a) cantilever and (b) doubly clamped beam with dielectrophoretic actuation.

force (deflection) generated for given voltage applied is typically smaller than in the case of the simple electrostatic actuation. For large static actuation, metals are placed near nanobeam in a slightly different fashion from the previous method as shown in Fig. 1(c). Most of the steps are similar except for the positioning of the electrode patterns. By making PMMA width slightly narrower than nanobeam width, evaporated metal is deposited both on the nanobeam and the bottom substrate. SEM image of the final device is shown in Fig. 2(c). Electrodes, one on the bottom, and the other on the nanobeam serve as driving electrodes. On the nanobeam, thin wire electrodes formed on either edge, so as to leave a large window for optical access to the color center. When a DC voltage is applied, the nanobeam is pulled down by electrostatic force, thereby applying strain to color centers embedded in diamond.

By using such an electrostatic MEMS device, we perform photoluminescence excitation (PLE) measurements on SiVs in the cantilever while deflecting the beam by applying DC voltage. The $\mathrm{SiV}$ is known to have two orbital states each in the ground and excited manifolds, and therefore its emission spectrum consists of four distinct lines [17]. Fig. 4 shows how the emission wavelengths of these four transitions depend on the applied voltage, in the case of the device shown in Fig. 2(c). The shifts in the emission wavelengths are entirely due to the applied strain since, due to the inversion symmetry, SiV does not show a linear Stark effect. Detailed analysis of electronic energy level shifts and the exact effect of strain are beyond the scope of the current paper, and will be presented elsewhere.

\section{DISCUSSION}

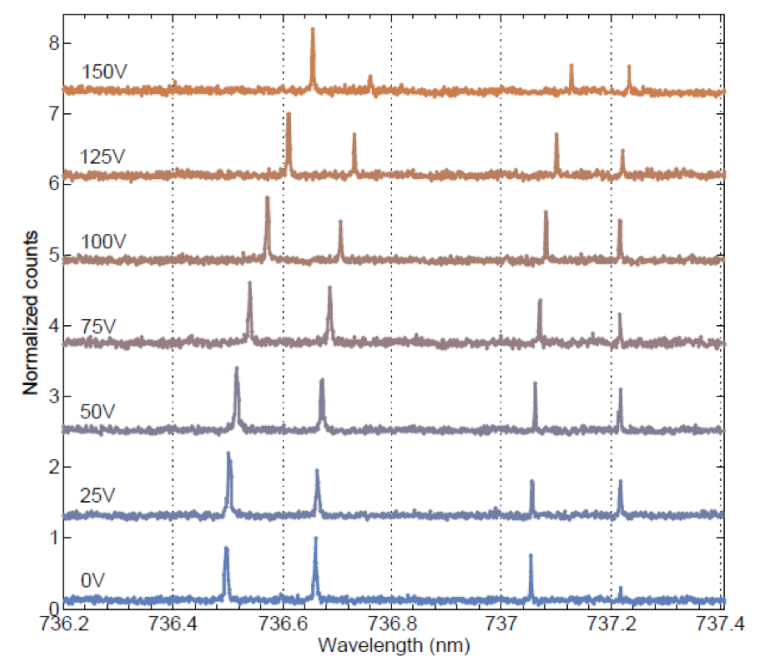

Figure 4: PLE spectra with varying applied voltage to the device shown in Fig. 2(c). Four distinct peaks reveal the electronic structure of a single $\mathrm{SiV}$. 
In this section, we discuss the design process for dielectrophoretic and electrostatic devices in the context of color center experiments. The dielectrophoretic scheme maintains high resonator $Q$ factor, which is desirable for applications relying on dynamic actuation of mechanical modes. In particular, this is important to achieve strong coupling between the qubit and the mechanical resonator. This condition can be quantified by a figure called the co-operativity, which needs to exceed $1[2,4]$.

$$
C=\frac{g^{2}}{n_{t h} \kappa \gamma}>1
$$

Here, $g$ is the coupling rate between the qubit levels due to a single phonon in the mechanical mode, $n_{t h}$ is the thermal phonon occupation of the mechanical mode of interest, $\kappa$ is the intrinsic mechanical damping rate (inversely proportional to $Q$ ), and $\gamma$ is the qubit-dephasing rate. A large coupling rate requires the smaller scale of the device in general $\left(g \propto \sqrt{\frac{1}{l^{3} w}}, l\right.$ is the length and $w$ is the width of the cantilever of rectangular cross section). Therefore, it is of great importance to achieve high $Q$ for small-scale devices, which is known to be difficult [18]. Due to the premature stage of diamond fabrication compared to silicon fabrication, options for on-chip SCD mechanical actuator are limited. The most obvious approach is to deposit a conducting film and use it for electrostatic actuation as in Fig. 2(c). However, it is well known that film deposition causes degradation in $Q$ [18]. Pursuing this goal is effectively similar to achieving high $f Q$ product, which is the fundamental figure of merit in MEMS. In contrast to the electrostatically actuated device, dielectrophoretic actuation scheme leaves the mechanical resonator intact from any foreign material, which helps to preserve $Q$. As a result, $f Q$ product of 6.8 $\times 10^{12}$ has been demonstrated, for $260 \mathrm{~nm}$ wide, $4 \mu \mathrm{m}$ long cantilever (Fig. 2(a)) whose resonance frequency is approximately $18.3 \mathrm{MHz}$ [14].

Although dielectrophoretic actuation has the above benefits, it is not ideal for static actuation due to its small force for a given voltage compared to electrostatic actuation. Therefore, electrostatic actuation is appealing for applications where high static strain is desired. To apply significant strain, application of high voltage and large deflection is required. However, a few fundamental and practical limitations need to be considered. First of all, as is always the case for the electrostatic actuation, pull-in instability occurs when the distance between two electrodes reaches a certain threshold. Pull-in instability can be simulated by
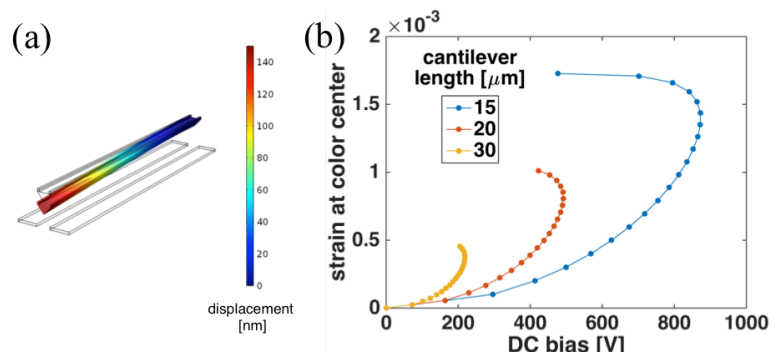

Figure 5: (a) Illustrations of the device with electrostatic actuation when $120 \mathrm{~V}$ is applied to $30 \mu \mathrm{m}$ long, $1.4 \mu \mathrm{m}$ wide diamond cantilever. (b) Simulated strain applied to color centers near the clamp of the cantilever. Turnaround points in the graph represent pull-in instabilities. using finite element method (FEM) and solving the inverse problem - i.e. for the given structure, voltage required to reach a target displacement is calculated. In Fig. 5(a), a 3D cartoon of the simulation is shown to illustrate the concept of the device. To have $150 \mathrm{~nm}$ tip displacement of $1.4 \mu \mathrm{m}$ wide, $830 \mathrm{~nm}$ thick and $30 \mu \mathrm{m}$ long diamond cantilever with the triangular cross section, approximately $120 \mathrm{~V}$ needs to be applied to this system. By sweeping target displacement and calculating corresponding voltage, displacement as a function of voltage can be plotted. Typically, pull-in instability manifests itself as a turnaround point in the plot. The FEM simulation gives strain tensor inside the cantilever as well, and it is a monotonically increasing function of the tip displacement. Such a plot is shown in Fig. 5(b). The device cross-section is the same as the one in Fig. 5(a), and the three different lengths are simulated. Before pull-in instability, longer (floppier) cantilevers give larger strain than shorter (stiffer) ones for a given voltage. When the voltage is increased, however, longer beams reach the pull-in instability at a smaller applied voltage. Thus, assuming that high enough dc voltage can be applied, shorter beams are desirable as they result in much larger achievable strain.

In reality, however, the large applied voltage and resulting electric field can lead to breakdown and a surge in current. This phenomenon is well characterized by the Paschen curve which expresses breakdown voltage as a function of pressure and the gap product [19]. It was explained that breakdown is a result of avalanche ionization of atoms between the gap caused by accelerated electrons [20]. However, in nanoscale devices, where the mean free path of the electron is larger than the gap, avalanche ionization is likely not the breakdown mechanism. Under this condition, if the electric field is strong enough to overcome the work function of the metal, it can directly pull out electrons through a process called field emission. In practice, the deposited film is not entirely smooth and hence field emission induced current can locally vaporize the metal, due to intensified heat [21].

In addition to breakdown due to the above phenomena, nonzero surface current on the diamond and resultant destruction of the metal need to be considered. Although diamond is an excellent insulator, a non-zero current through this device (ranging from $<\mathrm{nA}$ to hundreds of $\mu \mathrm{A}$ depending on the conditions) is always measured in our experiment. Typically, for many insulators, moisture adsorption on the surface and resulting electrochemical migration causes a small current [22]. At an applied voltage of $50 \mathrm{~V}$ or below, this current is relatively small (on the order of pA) and does not destroy nano-scale electrodes. However, at a higher voltage, on the order of hundreds of volts, current can grow fast and eventually cause fusing or bursting of small electrodes [23]. These two limiting factors, namely dielectric breakdown, and surface currents dictate the practical upper limit on the dc voltage used in these devices. Within these limits, one can use pull-in instability simulation results to design the dimensions of the cantilever that maximizes the applied strain.

Another point worthy of the discussion is the stress caused by metal film deposition. In principle, when a thin film is deposited on the substrate, it may have compressive or tensile residual stress depending on various factors such as film thickness, substrate temperature. Although the residual stress from the thin film can complicate the strain-tuning experiments with color centers and compromise the strength of the applied strain, its adverse effects can be minimized by using ultra-low film thickness at the cost of additional processing layers. In contrast, it can even aid the experiment if the system is cleverly designed [24].

\section{CONCLUSIONS}




\section{Summary}

In summary, we have demonstrated a SCD fabrication scheme for MEMS/NEMS devices by combining angled-etching and the conventional lift-off processes. MEMS/NEMS devices compatible with color center experiments with different requirements are realized with careful design of electrode patterns. In particular, dynamic actuation is demonstrated via dielectrophoretic forces, and static actuation is demonstrated via electrostatic forces. Dielectrophoretic actuation is useful to maintain high fQ product in small scale devices, while electrostatic actuation is suitable for generation of high static strain. We show the application of static strain generation in $\mathrm{SiV}$ electronic level tuning, and discuss the design of this MEMS device within fundamental and practical limits.

\section{Outlook}

Our fabrications method can be potentially applied to make nanodevices in materials that lack high-quality thin film technology. For example, we have demonstrated angled-etching in quartz substrate as a proof of the concept, since its piezoelectric and electro-optical properties can be useful [9]. Furthermore, it may be applied to other popular materials with high-quality color centers, such as $4 \mathrm{H}-\mathrm{SiC}$.

\section{REFERENCES}

[1] S. D. Bennett, N. Y. Yao, J. Otterbach, P. Zoller, P. Rabl and M. D. Lukin, "Phonon-induced spin-spin interactions in diamond nanostructures: application to spin squeezing", Physical Review Letters, 110, 156402 (2013).

[2] P. Ovartchaiyapong, K. W. Lee, B. A. Myers and A. C. B. Jayich, "Dynamic strain-mediated coupling of a single diamond spin to a mechanical resonator", Nature Communications, 5, (2014).

[3] J. Teissier, A. Barfuss, P. Appel, E. Neu and P. Maletinsky, "Strain Coupling of a Nitrogen-Vacancy Center Spin to a Diamond Mechanical Oscillator", Physical Review Letters, 113, 020503 (2014).

[4] S. Meesala, Y.-I. Sohn, H. A. Atikian, S. Kim, M. J. Burek, J. T. Choy and M. Loncar, "Enhanced Strain Coupling of Nitrogen-Vacancy Spins to Nanoscale Diamond Cantilevers", Physical Review Applied, 5, 034010 (2016).

[5] E. R. MacQuarrie, T. A. Gosavi, N. R. Jungwirth, S. A. Bhave and G. D. Fuchs, "Mechanical Spin Control of NitrogenVacancy Centers in Diamond", Physical Review Letters, 111, 227602 (2013).

[6] D. A. Golter, T. Oo, M. Amezcua, K. A. Stewart and H. Wang, "Optomechanical Quantum Control of a Nitrogen Vacancy Center in Diamond", arXiv.org, quant-ph, (2016).

[7] M. J. Burek, N. P. de Leon, B. J. Shields, B. J. M. Hausmann, Y. Chu, Q. Quan, A. S. Zibrov, H. Park, M. D. Lukin and M. Loncar, "Free-Standing Mechanical and Photonic Nanostructures in Single-Crystal Diamond", Nano Letters, 12, 6084 (2012).

[8] B. J. M. Hausmann, M. Khan, Y. Zhang, T. M. Babinec, K. Martinick, M. McCutcheon, P. R. Hemmer and M. Loncar, "Fabrication of diamond nanowires for quantum information processing applications", Diamond \& Related Materials, 19, 621 (2010).
[9] P. Latawiec, M. J. Burek, Y.-I. Sohn and M. Loncar, "Faraday cage angled-etching of nanostructures in bulk dielectrics", Journal of Vacuum Science \&amp; Technology B, Nanotechnology and Microelectronics: Materials, Processing, Measurement, and Phenomena, 34, 041801 (2016).

[10] M. J. Burek, D. Ramos, P. Patel, I. W. Frank and M. Loncar, "Nanomechanical resonant structures in single-crystal diamond", Applied Physics Letters, 103, 131904 (2013).

[11] M. J. Burek, Y. Chu, M. S. Z. Liddy, P. Patel, J. Rochman, S. Meesala, W. Hong, Q. Quan, M. D. Lukin and M. Loncar, "High quality-factor optical nanocavities in bulk singlecrystal diamond.", Nature Communications, 5, 5718 (2014).

[12] ESPACER ${ }^{\text {TM }}$ - Showa Denko. (2012). Retrieved April 12,2016 , from

http://www.sds.com.sg/products/electronics/espacer/

[13] Q. P. Unterreithmeier, E. M. Weig and J. P. Kotthaus, "Universal transduction scheme for nanomechanical systems based on dielectric forces", Nature, 458, 1001 (2009).

[14] Y.-I. Sohn, M. J. Burek, V. Kara, R. Kearns and M.

Loncar, "Dynamic actuation of single-crystal diamond nanobeams", Applied Physics Letters, 107, 243106 (2015).

[15] K. L. Ekinci, "Electromechanical Transducers at the Nanoscale: Actuation and Sensing of Motion in

Nanoelectromechanical Systems (NEMS)", Small, 1, 786 (2005).

[16] B. D. Hauer, C. Doolin, K. S. D. Beach and J. P. Davis, "A general procedure for thermomechanical calibration of nano/micro-mechanical resonators", Annals of Physics, condmat.mes-hall, (2013).

[17] H. Sternschulte, K. Thonke, R. Sauer and P. C. Münzinger, "1.681-eV luminescence center in chemical-vapordeposited homoepitaxial diamond films", Physical Review B, 50, 14554 (1994).

[18] M. Imboden and P. Mohanty, "Dissipation in nanoelectromechanical systems", Physics Reports, 534, 89 (2014).

[19] F. Paschen, "Ueber die zum Funkenübergang in Luft, Wasserstoff und Kohlensäure bei verschiedenen Drucken erforderliche Potentialdifferenz", Annalen der Physik, 273, 69 (1889).

[20] J. S. Townsend, Electricity in gases, Рипол Классик,

1915.

[21] F. W. Strong, J. L. Skinner, P. M. Dentinger and N. C. Tien. "Electrical breakdown across micron scale gaps in MEMS structures". MOEMS-MEMS 2006 Micro and Nanofabrication, San Jose, CA, USA, International Society for Optics and Photonics, (2006), p. 611103

[22] S. Zhan, M. H. Azarian and M. G. Pecht, "Surface Insulation Resistance of Conformally Coated Printed Circuit Boards Processed With No-Clean Flux", IEEE Transactions on Electronics Packaging Manufacturing, 29, 217 (2006).

[23] K. C.-Y. Chen, L. K. Warne, Y. T. Lin, R. L. Kinzel, J. D. Huff, M. B. McLean, M. W. Jenkins and B. M. Rutherford, "Conductor fusing and gapping for bond wires", Progress In Electromagnetics Research M, 31, 199 (2013).

[24] S. Han, T. J. Seok, N. Quack, B.-W. Yoo and M. C. Wu, "Large-scale silicon photonic switches with movable directional couplers", Optica, 2, 370 (2015).

\section{CONTACT}

* M. Lončar, tel: +1-617-495-5798; loncar@seas.harvard.edu 